\title{
HELMINTOS GASTROINTESTINALES DE CARPINCHOS (Hydrochoerus hydrochaeris) EN SISTEMA DE CRIANZA SEMI-INTENSIVO EN LA REGIÓN SUR DEL ESTADO DE RIO GRANDE DO SUL, BRASIL
}

\author{
WENDT, Luciana Welter ${ }^{3}$ \\ MÜLLER, Gertrud ${ }^{2}$; \\ PINHEIRO, Max ${ }^{5}$; \\ SANTOS, Laura Maria Jorge de Faria ${ }^{6}$; \\ SILVA, Maria Antonieta Machado Pereira da ${ }^{2}$; \\ GONZALES, Hermann Grau ${ }^{4}$; \\ RUAS, Jerônimo Lopes ${ }^{1}$; \\ FARIAS, Nara Amélia ${ }^{2}$.
}

Recebido: 01/04/2016

Aceito: 03/08/2016

'Laboratório Regional de Diagnóstico, Faculdade de Veterinária (FAVET), Universidade Federal de Pelotas (UFPEL), Brasil; ${ }^{2}$ Departamento de Microbiologia e Parasitologia, Instituto de Biologia (IB), UFPEL; ${ }^{3}$ Programa de Pós-Graduação em Veterinária, FAVET, UFPEL; ${ }^{4}$ Veterinario UTU - Consejo de Educación Técnico Profesional Rivera, Uruguay; ${ }^{5}$ Investigador EMBRAPA-Clima Temperado Pelotas, Brasil; ${ }^{6}$ Programa de Pós-Graduação em Parasitologia, IB, UFPEL.

\section{RESUMEN}

os carpinchos son roedores silvestres, cuya crianza intensiva y semi-intensiva, destinadas al consumo humano, han tenido un creciente aumento, dada toda la calidad de su carne. Treinta y cuatro carpinchos (13 cachorros, con edades inferiores a seis meses, y 21 adultos) de crianza semi-intensiva en el municipio de Pelotas, sur del estado de Rio Grande do Sul, fueron abatidos y sus helmintos gastrointestinales, investigados. Para los cachorros se encontraron los siguientes helmintos con sus respectivas prevalencias y carga parasitaria: Capillaria hydrochoeri $(46,15 \%$ e 11,5 especímenes), Hydrochoerisnema anomalobursata $(76,92 \%$ e 34,2 especímenes), Protozoophaga obesa (46,15\% y 6,33 especímenes) y Monoecocestus hagmanni (23,07\%y 1,66 especímenes). En carpinchos adultos habían cinco especies: $C$. hydrochoeri (66,66\% y 16,42 especímenes), $H$. anomalobursata (42,85\% y 79,88 especímenes), P. obesa ( $100 \%$ y $1.655,42$ especímenes), $M$. hagmanni $(14,28 \%$ y 38,33 especímenes) y Monoecocestus macrobursatum (4,76\% y dos ejemplares). M. hagmanni y M. macrobursatum, parasitando carpinchos en Rio Grande do Sul (RS) son nuevos registros.

Palabras clave: Parásitos gastrointestinales. Prevalencia. Nematodos. 


\section{INTRODUCCIÓN}

Los carpinchos (Hydrochoerus hydrochaeris, Linnaeus, 1766) son los mayores roedores existentes actualmente en el mundo, llegando a pesar $100 \mathrm{Kg}$. El $\mathrm{H}$. hydrochaeris se encuentra en el este de Colombia, en los Llanos Orientales, en los llanos de Venezuela, en Surinam, en Guyana y en Guyana Francesa. Lo mismo que en las regiones amazónicas de Ecuador, Perú, Bolivia, Argentina y Brasil (ALHO et. al, 1987). Por lo tanto, geográficamente corresponde a las cuencas hidrográficas de los principales ríos sudamericanos. El carpincho posee potencial económico debido a la demanda por cuero, carne y grasa. Además, posee gran rusticidad, eficiencia reproductiva y se alimenta exclusivamente de voluminosos, no compitiendo directamente con el hombre. Esas características explican su potencial para la explotación comercial (CATTO, 2000). Las enfermedades parasitarias son una de las principales causas de mortalidad de carpinchos, principalmente en animales criados en altas densidades y bajo condiciones de estrés (SILVA NETO, 1995), se ha relacionado con la presencia de los helmintos Monoecocestus macrobursatum, Strongyloides chapini, Capillaria hydrochoeri y Protozoophaga obesa (BONUTI et al., 2002). En Brasil se relató la presencia de Hydrochoerisnema anomalobursata, Vianella hydrochoeri, Haemonchus sp., Trichostrongylus axei, Cooperia punctata, Cooperia pectinata, Taxorchis schistocotyle, Hippocrepis hippocrepis (ARANTES, 1983; MATTOS JUNIOR; SANAVRIA, 1993; NASCIMENTO et al. 1991). Sinkoc (1997) registró M. hydrochoeri, T. schistocotyle, H. hippocrepis, Strongyloides sp., Trichuris spp., C. hydrochoeri, V. hydrochoeri, H. anomalobursata, P. obesa, Monoecocetus jacobi y Neocladorchis cabrali parasitando el tracto gastrointestinal de carpinchos de vida libre en el sur de Rio Grande do Sul en la región de Banhado do Taim. Como la mayoría de las publicaciones citan helmintos en animales de vida libre, el presente estudio tuvo como objetivo presentar los registros de las especies de helmintos parásitos de carpinchos criados en sistemas semi-intensivos, en la región sur de Rio Grande do Sul. 


\section{MATERIAL Y MÉTODOS}

Fueron colectados los contenidos gastrointestinales de 34 carpinchos criados en sistema semi-intensivo en el municipio de Pelotas, sur del estado de Rio Grande do Sul, Brasil. De estos, 13 eran cachorros con promedio de peso de 3,30 Kg, y 21 adultos con promedio de peso de 33,55 $\mathrm{Kg}$. A los cachorros se les analizó todo el contenido gastrointestinal luego de la necropsia. De los adultos, a cuatro se les hizo la necropsia y los restantes 17 fueron abatidos en un frigorífico de la ciudad de Pelotas (RS). Luego de retirado el tracto gastrointestinal, se hicieron ligaduras dobles aislando las porciones de estómago, intestino delgado, ciego y colon recto. La colecta y el procesamiento de los helmintos fue realizada de acuerdo al método descrito por Sinkoc (1997). La identificación taxonómica a nivel genérico se efectuó acorde con Travassos (1937), Amato y Amato (1993) y Gibbons (2010). El material está depositado en la colección biológica del Instituto de Biologia de la Universidade Federal de Pelotas. Los cálculos para estimar los parámetros se realizaron según Bush et al. (1997). Los valores individuales fueron tabulados en hojas de cálculo y los parámetros analizados de acuerdo con la categoría a través del programa Statistix R. Para el análisis de prevalencia se aplicó el test de Qhi-cuadrado (X2) siendo sustituido por el Test Exacto de de Fisher cuando los valores esperados eran menos de cinco.

\section{RESULTADOS Y DISCUSIÓN}

En este estudio fueron encontradas cinco especies de helmintos, tres especies de nematodos Capillaria hydrochoeri (Enoplida, Trichuridae), H. anomalobursata (Strongyloidea, Trichostrongylidae), P. obesa (Oxyuroidea, Oxyuridae) y dos especies de cestodos, M. hagmannie y M. macrobursatum (Cyclophyllidea, Anoplocephalidae). Los helmintos se encontraron parasitando el tracto intestinal de los carpinchos (Tablas 1 y 2 ). 
Tabla 1 - Prevalencia y localización en el tracto gastrointestinal en carpinchos cachorros ( $n=13)$, adultos ( $n=21)$ y general $(n=34)$ en crianza semi-intensiva en la región sur de Rio Grande do Sul, Brasil.

Órgano/helminto

Estómago

Capillaria hydrochoeri

Hydrochoerisnema anomalobursata

Intestino delgado

Capillaria hydrochoeri

Hydrochoerisnema anomalobursata

Monoecocestus hagmanni

Monoecocestus macrobursatum

Ciego

Protozoophaga obesa

Colon/ recto

Protozoophaga obesa

30,76

52,38

23,80

15,38

20,58

$\begin{array}{lll}46,15 & 52,38 & 50,00 \\ 76,92 & 33,33 & 50,00 \\ 23,07 & 14,28 & 17,64 \\ 0 & 4,76 & 2,94\end{array}$

46,15

100

79,41

En el estómago, en $58,8 \%$ de los carpinchos fueron encontradas C. hydrochoeri en 15 animales e $H$. anomalobursata en siete animales. Dos carpinchos estaban parasitados simultáneamente por las dos especies. En el intestino delgado, 88,2\% de los carpinchos se encontraron parasitados por uno a tres especímenes de los siguientes hemintos: $C$. hydrochoeri, $H$. anomalobursata, M. hagmanni y M. macrobursatum. En el ciego y en el segmento colon/recto hubo parasitismo monoespecífico, siendo que 27 carpinchos (79,4\%) presentaron P. obesa y C. hydrochoeri Travassos, 1916 Sinonimo: Echinocoleus hydrochoeri Moravec, 1982, respectivamente. Los cachorros no presentaron diferencias en cuanto a la prevalencia $(p=0,23)$ e intensidad de infección $(p=0,12)$, en relación a los adultos.

En carpinchos de vida libre, durante la época seca, los animales se concentran en torno a aguadas, y en la época de lluvias ocurre dispersión de esos animales. Las condiciones de crianza en cautiverio, en espacio reducido, no permiten esa dispersión natural, lo que 
favorece el encuentro parásito/hospedador y una mayor carga parasitaria (MAYAUDON TARBES, 1980). C. hydrochoeri Travassos, 1916 Sinónimo: E. hydrochoeries ha sido citado en diferentes regiones de Brasil parasitando carpinchos en ambiente natural y en cautiverio (BONUTI et al., 2002; COSTA; CATTO, 1994; SINKOC et al., 2004, SINKOC et al., 2009). C. hydrochoeri es parásito del estómago de carpinchos adultos y del intestino delgado de carpinchos jóvenes y adultos en ambiente natural, en la región de Taim (RS) (SINKOCK, 1997). El autor también observó prevalencia superior e intensidad de infección inferior, en los carpinchos adultos, en relación a los jóvenes. En tanto, en un trabajo realizado por Sinkock et al. (2004) en un sistema de crianza semi-intensivo en el municipio de Araçatuba (SP), se observó una prevalencia similar (58,33\%) y diferente intensidad promedio entre animales jóvenes y adultos $(70,00$ y 45,71$)$, respectivamente.

Tabla 2 - Intensidad de la infección por helmintos gastrointestinales en carpinchos cachorros ( $\mathrm{n}=13$ ), adultos $(n=21)$ y general $(n=34)$ en crianza semi-intensiva en la región sur de Rio Grande do Sul, Brasil.

Órgano/helminto Intensidade de infección (especimenes)

Cachorros Adultos General

Estómago

Capillaria hydrochoeri

5,25

7

Hydrochoerisnema anomalobursata

5,5

11,4

9,71

Intestino delgado

Capillaria hydrochoeri

8

13,63

11,64

Hydrochoerisnema anomalobursata

33,2

94,57

58,47

Monoecocestus hagmanni

1,66

38,33

20

Monoecocestus macrobursatum

0

2

2

Ciego

Protozoophaga obesa

6,33

1484,09

1155,70

Colon/ recto

Protozoophaga obesa

0

170,85

170,85 
Los registros de presencia de $H$. anomalobursata se limitan, en Brasil, en la región del Pantanal (BONUTI et al., 2002), en Rio de Janeiro (MATTOS JUNIOR; SANAVRIA, 1993), y en Rio Grande do Sul (SINKOC et al., 2009). En el presente estudio, este parásito fue encontrado en el estómago e intestino delgado de cachorros y adultos, con parámetros que no difirieron estadísticamente. La infección por $H$. anomalobursata, trichostrongílido el cual produce lesiones en la mucosa gastrointestinal, puede influenciar la capacidad de absorción de nutrientes (ARANTES, 1983; MATTOS JUNIOR.; SANAVRIA, 1993). Por eso es importante el monitoreo de los animales a través de exámenes coprológicos periódicos, ya que, en un sistema de explotación económica, la ganancia de peso es fundamental. Mattos Junior et al. (1996) citan la presencia de edema, erosiones e infiltrado inflamatorio en el intestino delgado de carpinchos jóvenes causados por $H$. anomalobursata. Los autores también relataron, en la necropsia de un carpincho en cautiverio, numerosas lesiones en la pared del intestino delgado como erosiones, edema e infiltrados inflamatorios asociados a la presencia de 500 ejemplares de $H$. anomalobursata adheridos a la mucosa. En ambiente natural Sinkock (1997) encontró este parásito en el estómago y en el intestino delgado de carpinchos, con intensidad de infección superior en los jóvenes con relación a los adultos. Con todo, Sinkock et al. (2009), observaron la misma prevalencia (58,33\%) del parásito, independiente de la edad del hospedador, en la región del Taim (RS). En carpinchos criados intensivamente, Ribeiro y Amato (2003) demonstraron una prevalencia de $60 \%$ e intensidad promedio de infección de 371,11 especimenes de $H$. anomalobursata en el intestino delgado. $P$. obesa puede ser un importante competidor, pues se alimenta fundamentalmente de protozoarios simbiontes del ciego, y presenta alta intensidad promedio de infección (NOGUEIRA; CRUZ, 2007). Según Costa y Catto (1994) las lesiones asociadas a altas infecciones por el parásito, pueden interferir en el proceso digestivo del carpincho, causando reducción en la capacidad de conversión alimenticia ya que se alimenta básicamente de la microbiota intestinal. En este estudio, el parásito presentó parámetros significativamente superiores en los carpinchos adultos en relación a los cachorros $(p=0,05)$. Las tasas de prevalencia e intensidad de infección, fueron, en adultos, $100 \%$ y 1655,42 especímenes, mientras que, en jóvenes, fueron del $46,15 \%$ y 6,33 especímenes. En ambiente natural, Sinkock et al. (2009) observaron parasitismo superior en carpinchos jóvenes con 
relación a los adultos. Ribeiro (2002) observó prevalencia de 93,3\% e intensidad promedio de infección de 2493,97 especímenes, en el ciego de carpinchos adultos, índices semejantes a los encontrados por Sinkock et al. (2009) (95,83\%) y superiores a los encontrados por Nascimento et al. (1991) (66,66\%).

M. hagmanni, ya fue citado en el Brasil, en carpinchos de vida libre en Rio de Janeiro y en la región del Pantanal (BONUTI et al., 2002; COSTA; CATTO, 1994), y en cautiverio en el estado de São Paulo (SINKOC et al., 2004). En el presente estudio no hubo diferencia estadística significativa entre los parámetros de prevalencia $(p=0,65)$ e intensidad de infección $(p=0,69)$ entre cachorros y adultos. Este es el primer registro de $M$. hagmanni en el estado de Rio Grande do Sul. En el presente estudio, dos ejemplares de M. macrobursatum fueron colectados del intestino delgado de un carpincho. En el Brasil, hay registros de este parásito solo en carpinchos de la región del Pantanal (BONUTTI et al., 2002). Sinkock et al. (2009) encontraron dos especies del género: Monoecocestus hydrochoeri y M. jacobi, más prevalentes en jóvenes. Los carpinchos examinados en este estudio recibían periódicamente antiparasitarios, lo que puede haber influenciado los parámetros, principalmente la abundancia y la intensidad promedio de parasitismo. Helmintos de la Clase Trematoda no fueron observados, posiblemente debido a la ausencia de infección en los carpinchos padres, que inicialmente formaron la manada (NOGUEIRA; CRUZ, 2007). Los carpinchos presentan un comportamiento acuático pudiendo transformar el agua en un medio de diseminación de patógenos. El número de carpinchos criados en cautiverio viene aumentando, lo que torna importante el conocimiento de los parásitos que atacan a esta especie de roedor. El manejo sanitario en cautiverio se inicia con la identificación de individuos que presenten menores niveles de estrés bajo condiciones artificialmente impuestas, en la adecuación del ambiente a la especie y en el manejo nutricional de los animales asegurando mayor resistencia a las enfermedades. Los resultados obtenidos revelan que la crianza de esa especie, con fines económicos requiere un riguroso control sanitario que contemple las parasitosis gastrointestinales. La presencia de estos parásitos puede comprometer el rendimiento productivo de los animales. El presente trabajo registra por primera vez la composición helmíntica de carpinchos en el estado de Rio Grande do Sul en animales en sistema de crianza semi-intensivo. 


\section{CONCLUSION}

Los carpinchos en crianza semi-intensiva en sur del estado de Rio Grande do Sul son parasitados por los siguientes parásitos: Animales jóvenes por Capillaria hydrochoeri, Hydrochoerisnema anomalobursata, Protozoophaga obesa y Monoecocestus hagmanni. En animales adultos C. hydrochoeri, H. anomalobursata, P. obesa, M. hagmanni y Monoecocestus macrobursatum. M. hagmanni y M. macrobursatum parasitando carpinchos en Rio Grande do Sul son nuevos registros.

\section{HELMINTOS GASTRINTESTINAIS DE CAPIVARAS (Hydrochoerus hydrochaeris) EM CRIAÇÃO SEMI-INTENSIVA NA REGIÃO SUL DO ESTADO DO RIO GRANDE DO SUL, BRASIL}

\section{RESUMO}

C

apivaras são roedores silvestres, cuja criação intensiva e semi-intensiva, destinadas ao consumo humano, tiveram um aumento crescente em razão da qualidade da carne. Trinta e quatro capivaras (13 jovens, com menos de seis meses de idade, e 21 adultos) de uma criação semi-intensiva no município de Pelotas, sul do estado do Rio Grande do Sul, foram abatidas e investigados seus helmintos gastrintestinais. Em animais jovens foram encontrados os seguintes helmintos com as respectivas prevalências e carga parasitária: Capillaria hydrochoeri (46,15\% e 11,5 espécimes), Hydrochoerisnema anomalobursata (76,92\% e 34,2 espécimes), Protozoophaga obesa (46,15\% e 6,33 espécimes) e Monoecocestus hagmanni $(23,07 \%$ e 1,66 espécimes). Em capivaras adultas foram encontradas cinco espécies: $C$. hydrochoeri (66,66\% e 16,42 espécimes), $H$. anomalobursata (42,85\% e 79,88 espécimes), $P$. obesa (100\% e 1655,42 espécimes) $M$. hagmanni (14,28\% e 38,33 espécimes) e Monoecocestus macrobursatum (4,76\% e duas espécimes). $M$. hagmanni e M. macrobursatum, parasitando capivaras no Rio Grande do Sul são novos registros.

Palavras-chave: Parasitos gastrintestinais. Prevalência. Nematoides. 


\section{GASTROINTESTINAL HELMINTHS OF CAPYBARAS (Hydrochoerus hydrochaeris) IN SEMI-INTENSIVE BREEDING SYSTEM IN THE SOUTHERN REGION OF RIO GRANDE DO SUL STATE, BRAZIL}

\section{ABSTRACT}

C apybaras are wild rodents, whose intensive and semi-intensive breeding have increased for human feeding because of the quality of their meat. Thirty-four capybaras (13 young capybaras with less than six months of age, and 21 adults) from a semi-intensive breeding system in the city of Pelotas, southern state of Rio Grande do Sul, were slaughtered and their gastrointestinal helminthes were investigated. In young animals the following helminths were found with the respective prevalence and parasite load: Capillaria hydrochoeri (46.15\% and 11.5 specimens), Hydrochoerisnema anomalobursata (76.92\% and 34.2 specimens), Protozoophaga obesa $(46.15 \%$ and 6.33 specimens) and Monoecocestus hagmanni (23.07\% and 1.66 specimens). In adult capybaras there were five species: C. hydrochoeri (66.66\% and 16.42 specimens), $H$. anomalobursata $(42.85 \%$ and 79.88 specimens), $P$. obesa ( $100 \%$ and 1655.42 specimens) M. hagmanni ( $14.28 \%$ and 38.33 specimens) and Monoecocestus macrobursatum (4.76\% and two specimens). M. hagmanni and M. macrobursatum parasitizing capybaras in Rio Grande do Sul are new records.

Keywords: Gastrointestinal parasites. Prevalence. Nematodes.

\section{REFERENCIAS}

ALHO, C. J. R.; CAMPOS, Z. M.; GONÇALVES, H. C. Ecologia de Capivara (Hydrochoerus hydrochaeris, Rodentia) do Pantanal: I Habitats, densidades e tamanho de grupo. Revista Brasileira de Biologia, v. 47, p. 87-97, 1987.

AMATO, S. B.; AMATO, J. F. R. A new species of Phyllodistomum Braun, 1899 (Digenea: Gorgoderidae) from Rhamdia quelen (Quoy e Gaimard, 1824) (Siluriformes: Pimelodidae). Memórias do Instituto Oswaldo Cruz, v. 88, p. 557-559, 1993.

ARANTES, I. G. Considerações sobre Trichostrongyloidea Cram, 1927, parasitas de capivaras (Hydrochaeris hydrochaeris hydrochaeris Linnaeus, 1766) provenientes do Estado do Mato Grosso do Sul (Municípios de Angélica, Aquidauana e Rio Verde). Jaboticabal: UNESP, 1983, 76p. Tese (Livre Docência), Faculdade de Ciências Agrárias e Veterinárias, Universidade Estadual Paulista, 1983. 
BONUTI, M. R.; NASCIMENTO, A. A.; MAPELI, E. B.; ARANTES; I. G. Helmintos gastrintestinais de capivaras (Hydrochoerus hydrochaeris) na sub-região de Paiaguas, Pantanal do Mato Grosso do Sul, Brasil. Semina: Ciências Agrárias, v. 23, n. 1, p. 57-62, 2002.

BUSH, A. O; LAFFERTY, K. D; LOTZ, J. M; SHOSTAK, A. W. Parasitology meets ecology on its own terms: Margolis et al. revisited. Journal of Parasitology, v. 83, p. 575-583, 1997.

CATTO, B. C. Endoparasitos de animais domésticos e silvestres do Pantanal: Helmintos, Acantocéfalos, Pentastomídeos e Protozoários. In: SIMPÓSIO SOBRE RECURSOS NATURAIS E SÓCIO-ECONÔMICOS DO PANTANAL, OS DESAFIOS DO NOVO MILÊNIO, 3, 2000, Corumbá. ANAIS. Corumbá: Embrapa, p. 1-17, 2000. Disponível em: <http://www.cpap.embrapa.br/agencia/congresso/Bioticos/CATTO-088.pdf> Acesso em: 09 maio 2014.

COSTA, C. A. F.; CATTO, J. B. Helmintos parasitos de capivaras (Hydrochaeris hydrochaeris) na sub-região da Nhecolândia, Pantanal-sul-matogrossense. Revista Brasileira de Biologia, $v$. 51, p. 39-48,1994.

GIBBONS, L. M. Keys to the Nematode Parasites of Vertebrates. Supplementary volume. Wallingford (UK): CAB International, 2010. 416p.

MATTOS JUNIOR, D. G.; SANAVRIA, A. Helmintos parasitos de capivaras (Hydrochaeris hydrochaeris) em sistema de criação de cativeiro, no estado do Rio de Janeiro. In: SEMINÁRIO BRASILEIRO DE PARASITOLOGIA VETERINÁRIA, 8, 1993, Londrina. ANAIS. Londrina: Colégio Brasileiro de Parasitologia Veterinária, 1993. p. 34.

MATTOS JUNIOR, D. G.; SANAVRIA, A.; TARTELLY, R. Ocurrence of Hydrochoerisnema anomalobursata Arantes \& Artigas, 1980 (Trichostrongyloidea) in capybaras (Hydrochoerus hydrochaeris) reared in semi-confinement in Rio de Janeiro State, Brazil. Parasitologia al Dia. v. 20, n. 1-2, p. 63-65, 1996.

MAYAUDON TARBES, H. Sobre una coleccion de helmintos del chiguire (Hidrochoerus hydrochoeris) de Venezuela. Revista de la Facultad de Ciencias Veterinarias, v. 28, n. 1-8, p. 19-47,1980.

NASCIMENTO, A. A.; TEBALDI, J. H.; ASCARI, H.; ARANTES, I. G. Helmintos parasitos de Hydrochoerus hydrochaeris hidrochaeris (Linnaeus, 1766) no estado do Mato Grosso do Sul, Brasil. Revista Brasileira de Parasitologia Veterinária, v. 1, p. 49, 1991.

NOGUEIRA, M. F.; CRUZ, T. F. Doenças da Capivara. Corumbá: Embrapa Pantanal, 2007. 74p. Disponível em:

<http://www.cpap.embrapa.br/publicacoes/download.php?arq_pdf=Livro030>.

RIBEIRO, S. M. B. Helmintos do sistema digestivo de Hydrochaeris hydrochaeris (Linnaeus, 1766) criadas em sistema semi-intensivo no município de Santo Antônio da Patrulha, Rio 
Grande do Sul, Brasil. Porto Alegre: UFRGS, 2002. Dissertação (Mestrado em Veterinária). Universidade Federal do Rio Grande do Sul, 2002.

RIBEIRO, S. M. B.; AMATO, S. B. Descrição de estruturas associadas com a bolsa copuladora e cone genital de Hydrochoerisnema anomalobursata Arantes \& Artigas, 1980

(Trichostrongyloidea, Vianaiidae). Arquivos do Instituto Biológico, v. 70, p.165-167, 2003.

SILVA NETO, P. B. Criação racional e comercial de animais silvestres. Jornal SOS Mata Atllântica, v. 7, n. 2, p. 3, 1995.

SINKOC, A. L. Helmintos gastrintestinais e artrópodos parasitos de capivara Hydrochaeris hydrochaeris (Linnaeus, 1766) em área de exploração pecuária na região do banhado do Taim, município de Rio Grande, RS. Porto Alegre: UFRGS, 1997. Dissertação (Mestrado em Veterinária), Faculdade de Medicina Veterinária, Universidade Federal do Rio Grande do Sul, 1997.

SINKOC, A. L.; BRUM, F. A.; MÜLLER, G.; BRUM, J. G. W. Helmintos Parasitos de Capivara (Hydrochoerus Hydrochaeris L. 1766) na Região de Araçatuba, São Paulo, Brasil. Arquivos do Instituto Biológico, n. 71, p. 329-333. 2004.

SINKOC, A. L.; BRUM, J. G. W.; MÜLLER, G. Gastrintestinal helminths of capybara (Hydrochoerus hydrochaeris, Linnaeus, 1766) in cattle breeding farm in the area of the Ecological Reserve of Taim, Rio Grande. Brazilian Archives of Biology and Technology v. 52, p. 327-333, 2009.

TRAVASSOS, L. Revisão da famíliaTrichostrongylidae Leiper. Memórias do Instituto Oswaldo Cruz, v. 1, p. 512, 1937. 